\title{
Clinical myopathy in patients with nephropathic cystinosis
}

\author{
Reza Sadjadi MD ${ }^{1}$ ( ) | Stacey Sullivan SLP ${ }^{2}$ | Natalie Grant BA ${ }^{1}$ | \\ Susan E. Thomas MD ${ }^{3}$ | Maya Doyle MSW, PhD, LCSW ${ }^{4}$ Colleen Hammond RN ${ }^{5}$ | \\ Rachel Duong BA ${ }^{1}$ | Camille Corre BS/BA ${ }^{1}$ | William David MD, $\mathrm{PhD}^{6}$ | \\ Florian Eichler $\mathrm{MD}^{1}$
}

${ }^{1}$ Center for Rare Neurological Diseases, Department of Neurology, Massachusetts General Hospital, Massachusetts

${ }^{2}$ Department of Neurology, Massachusetts General Hospital, Boston, Massachusetts

${ }^{3}$ Division of Pediatric Nephrology, Department of Pediatrics and Communicable Diseases, University of Michigan School of Medicine, Ann Arbor, Michigan

${ }^{4}$ Department of Social Work, School of Health Sciences, Quinnipiac University, Hamden, Connecticut

${ }^{5}$ Cystinosis Adult Care Excellence Initiative, Reading, Massachusetts

${ }^{6}$ Department of Neurology, Massachusetts General Hospital, Boston, Massachusetts

\section{Correspondence}

Reza Sadjadi, Center for Rare Neurological Diseases, Department of Neurology,

Massachusetts General Hospital, 55 Fruit

Street, Boston, MA 02114.

Email: rseyedsadjadi@partners.org

Funding information

This work was supported by a grant from the Cystinosis Research Foundation. None of the authors has any conflict of interest to disclose.

\begin{abstract}
Background: Nephropathic cystinosis is a lysosomal storage disorder. Patient survival years after renal transplantation has revealed systemic complications including distal myopathy and dysphagia.

Methods: We evaluated 20 adult patients with nephropathic cystinosis using patient-reported and clinical outcome measures. Standard motor measures, video fluoroscopy swallow studies, and tests of respiratory function were performed. We also used Rasch analysis of an initial survey to design a 16-item survey focused on upper and lower extremity function, which was completed by 31 additional patients. Results: Distal myopathy and dysphagia were common in patients with nephropathic cystinosis. Muscle weakness ranges from mild involvement of intrinsic hand muscles to prominent distal greater than proximal weakness and contractures.

Conclusions: In addition to further characterization of underlying dysphagia and muscle weakness, we propose a new psychometrically devised, disease specific, functional outcome measures for distal myopathy in patients with nephropathic cystinosis.

KEYWORDS

distal myopathy, dysphagia, nephropathic cystinosis, outcome measures, psychometrics
\end{abstract}

\section{1 | INTRODUCTION}

Nephropathic cystinosis is a rare autosomal recessive lysosomal storage disorder, due to mutations in the cystinosin gene (CTNS) located on chromosome $17 \mathrm{p} 13 .^{1-3}$ The basic defect is the impairment of the lysosomal membrane transport complex ${ }^{4,5}$ and free cystine accumulation in different tissues including kidney and muscles. The pathologic hallmark is deposition of cystine crystals and presence of autophagic, acid phosphatase positive

Abbreviations: 25-FW, timed 25-foot walk; 9-HPT, the 9-hole peg test; ACE, Adult Care Excellence Initiative; CTNS, cystinosin gene; DMFS, Distal Myopathy Function Scale; EAT-10, the 10-item eating assessment tool; FW, Timed 25-Foot Walk; IBM, inclusion body myositis; IBM-FRS, IBM-Functional Rating Scale; IQR, interquartile range; MDADI, The M. D. Anderson Dysphagia Inventory; MEP, maximum expiratory pressure; PAS, PenetrationAspiration Scale; PF, peak airflow; QOL, quality of life; RPCM, Rasch partial credit model; TUG, Timed Up and Go test; VFSS, video fluoroscopy swallow study. vacuoles in muscle tissues. Patients present with early failure to thrive ${ }^{6}$ and Fanconi syndrome. ${ }^{7,8}$ Systemic manifestations of cystinosis, such as myopathy, were not widely known before renal transplantation ${ }^{9,10}$ and introduction of beta-mercaptoethylamine (cysteamine) therapy. ${ }^{11,12}$ Cysteamine facilitates cystine transportation from lysosome through a different (intact) lysine transporter. ${ }^{13}$ Lower muscle and liver cystine levels were demonstrated in patients on long term cysteamine therapy. ${ }^{6,14-18}$ Nevertheless, unremitting accumulation of cystine, has led to more significant nonrenal morbidity and mortality. Many patients succumb to muscle weakness, ${ }^{19-21}$ swallowing difficulties, ${ }^{22}$ and aspiration. ${ }^{23}$

Unlike other lysosomal storage disorders, little is known about the pathophysiology of myopathy associated with nephropathic cystinosis. Muscle biopsy is often notable for abundant vacuoles and cystine crystals within perimyseal collagen fibrils. ${ }^{5}$ It has been speculated that distal 
myopathy is common in patients with cystinosis, even in the absence of clinically overt muscle weakness. ${ }^{16,20,21,24}$ Swallowing muscles are often involved and dysphagia is relatively common in patients with nephropathic cystinosis. ${ }^{18,22,25}$ Respiratory muscles are subsequently affected and respiratory muscle weakness can be life threatening. ${ }^{26}$

In 2016, an online survey was undertaken by the Adult Care Excellence Initiative (ACE), ${ }^{27}$ an advocacy group of individuals with cystinosis, their parents, clinicians, and researchers. ${ }^{28-30}$ The survey included multiple choice and associated open-ended questions related to physical health, medical care, treatment, psychological issues, social wellness, and financial burden. It was distributed online in English in two phases and received responses from adults with cystinosis from around the world. Through collaboration with the members of the ACE and access to the results of this surveys, we determined that swallowing difficulties and muscle weakness were pressing concerns that greatly affect the adult patient population.

A major obstacle in implementing clinical trials is the lack of information on evolution of symptoms and robust outcome measures and biomarkers that are sensitive and "responsive" enough to detect meaningful changes in disease status. In the current study, we sought to characterize muscle weakness and dysphagia in patients with nephropathic cystinosis and propose new disease-specific functional clinical outcome measures to better quantify disease progression in this patient population.

\section{2 | METHODS}

The study was approved by the research ethics committee, Partners Institutional Review Board at Massachusetts General Hospital. Informed consent was obtained in accordance with Institutional Review Board procedures.

We evaluated 20 adult patients with confirmed diagnosis of nephropathic cystinosis based on elevated leukocyte cystine levels, the presence of crystals in the cornea, or genetic mutation testing. We did not perform electrophysiologic evaluation or muscle biopsy to confirm presence of myopathic changes in our patient population. We believed evidence from prior pathology and electrodiagnostic studies were sufficient to assume that the weakness was a primarily myopathic process. ${ }^{5,16,20,24}$ Subjects were recruited by the Cystinosis Research Foundation and traveled to Massachusetts General Hospital in Boston for evaluation.

\section{1 | Clinical study}

Patients had neuromuscular examination, including manual muscle testing of proximal and distal upper and lower extremities, video fluoroscopy swallowing study (VFSS), and pulmonary function testing. Patients completed patient-reported measures (The M. D. Anderson Dysphagia Inventory $^{31}$ [MDADI] and The 10-item Eating Assessment Tool ${ }^{32}$ [EAT-10]) and clinical outcome measures (9-Hole Peg Test [9-HPT], Timed 25-Foot Walk [25-FW], Timed Up and Go test [TUG], and grip dynamometry).

\section{2 | Development of Distal Myopathy Function Scale}

A preliminary Distal Myopathy Function Scale (DMFS) focused on upper and lower extremity function was designed. ${ }^{33}$ Based on the feedback from the cystinosis ACE, and prior experiences with phenotypically similar conditions such as inclusion body myositis (IBM) ${ }^{31}$ and other distal myopathies ${ }^{31,32,34,35}$ we developed an item pool from recurring themes. The initial 22-item paper survey was administered during the clinical visit. We designed a 16-item survey based on results of the Rasch analysis of the initial survey. Thirty-one patients who did not participate in the study were independently contacted through the Cystinosis Research Foundation and completed the revised survey electronically using a REDCap survey tool.

\section{3 | Patient-reported outcomes}

The MDADI is a self-administered 20-item questionnaire designed to evaluate the impact of dysphagia on the quality of life (QOL) of patients with head and neck cancer, and adopted for this study of patients with nephropathic cystinosis. MDADI scores range between 20 and 100 with a higher score representing better day-to-day functional and better QOL.

The EAT-10 is a self-administered, symptom-specific outcome instrument for dysphagia. The EAT-10 scores range between 0 and 40 with higher scores representing more severe dysphagia. Normative data suggest that an EAT-10 score of 3 or higher is considered abnormal.

\section{4 | Clinical outcomes}

All patients were assessed by neurologists (R.S. and F.E.) who performed neurological evaluations including manual muscle testing (eye closure, mouth closure, neck flexion/extension, shoulder abduction/external rotation, elbow flexion/extension, wrist flexion/extension, finger extension/abduction, thumb abduction/flexion, deep finger flexors(II-IV), hip flexion/abduction/adduction/extension, knee flexion/extension, ankle dorsiflexion, plantar flexion, toe extension, deep toe flexion) and hand grip strength using a calibrated Jamar Hydraulic Hand Dynamometer. The TUG test was used to assess each patient's mobility, static and dynamic balance, walking ability, and fall risk. The $25-\mathrm{FW}$ was administered to assess quantitative mobility and leg function. The 9-HPT was used to measure finger dexterity.

A standardized VFSS was performed using three food textures of barium contrast (thin, nectar, and pudding). Each patient's VFSS was evaluated using the validated Penetration-Aspiration Scale (PAS), ${ }^{36}$ an 8-point ordinal scale of airway safety that describes the degree of airway invasion, the participant's response, and whether the invasive material is successfully ejected from the airway. We measured maximum expiratory pressure (MEP) and peak airflow (PF) for evaluation of respiratory function.

\section{5 | Statistical analysis}

We used R statistics software (version 3.2.2) for conventional analyses and Rasch partial credit model (RPCM) was performed using Winsteps software (3.92.1). We tabulated summary statistics for the clinical and patient-reported outcomes (Table 1). Nonparametric 
Wilcoxon-rank sum and chi-square trend tests were used to compare continuous and ordinal categorical outcomes between groups. Spearman rank correlations were used to assess the association between

TAB LE 1 Clinometric and patient-reported outcome measures in patients

\begin{tabular}{|llcc|}
\hline & Units & Mean $(95 \% \mathrm{Cl})$ & Median (IQR) \\
\hline MEP & $\mathrm{cmH}^{2} \mathrm{O}$ & $67.9(48.8 ; 87.0)$ & $68.5(31.0 ; 97.0)$ \\
\hline PF & $\mathrm{L} / \mathrm{min}$ & $247.3(190.8 ; 303.7)$ & $270.0(133.0 ; 328.5)$ \\
\hline Grip dynamometry & $\mathrm{KgF}$ & $23.1(14.7 ; 28.9)$ & $20.3(16.8 ; 26.5)$ \\
\hline 9-HPT & $\mathrm{s}$ & $23.3(20.5 ; 26.2)$ & $21.7(20.3 ; 25.2)$ \\
\hline TUG test & $\mathrm{S}$ & $7.0(6.3 ; 7.8)$ & $7.3(5.8 ; 8.2)$ \\
\hline 25-FW & $\mathrm{S}$ & $4.9(4.5 ; 5.4)$ & $5.1(3.9 ; 5.6)$ \\
\hline MDADI & - & $82.0(76.5 ; 87.5)$ & $84.5(72.5 ; 92.0)$ \\
\hline EAT-10 & - & $6.7(2.7 ; 10.7)$ & $2.5(0.5 ; 15.0)$ \\
\hline
\end{tabular}

Abbreviation: $\mathrm{Cl}$, confidence interval. responses. Cohen's suggested correlation co-efficient ( $r$ values) of less than 0.3 for mild, between 0.3 and 0.5 for medium, and greater than 0.5 for large correlations were used to define magnitude of association. ${ }^{37} \mathrm{P}$-value of less than 0.05 was considered significant.

RCPM was used to explore data for item-person targeting, item fitting, dependency, dimensionality and category response functioning. ${ }^{38}$ Rasch analysis compares the logarithmic probability of survey items (defined as item difficulty) with the total score by patients (defined as person ability). Comparing item difficulty range to person ability allows measuring relative sensitivity of items and exploring ceiling and floor effects (item-person targeting). In this model, items homogenously contribute to the total score to form a linear outcome measure. Rasch analysis estimates the homogeneity or "fitness" by comparing items (and individual respond thresholds) to an ideal linear model (fit statistics and dimensionality). ${ }^{39,40}$ Items with significant variability from the ideal linear model are examined to look for any similar patterns or trends suggestive of a dimensionality, or a concept beyond what the outcome measure is intended to capture as whole.

\begin{tabular}{|c|c|c|c|c|c|c|c|c|}
\hline & Age & Grip & 9-HPT & TUG & 25-FW & MDADI & EAT-10 & MEP \\
\hline Grip & 0.04 & - & & & & & & \\
\hline 9-HPT & -0.18 & -0.33 & - & & & & & \\
\hline TUG & -0.11 & -0.42 & $0.47 *$ & - & & & & \\
\hline $25-\mathrm{FW}$ & 0.01 & $-0.54 *$ & 0.38 & $0.85 * *$ & - & & & \\
\hline MDADI & -0.05 & 0.31 & -0.21 & -0.27 & -0.29 & - & & \\
\hline EAT-10 & 0.10 & -0.23 & -0.01 & 0.07 & 0.17 & $-0.82 * *$ & - & \\
\hline MEP & 0.27 & $0.55^{*}$ & -0.24 & $-0.61 * *$ & $-0.53^{*}$ & 0.20 & -0.12 & - \\
\hline PF & 0.10 & 0.37 & -0.45 & $-0.57 *$ & -0.35 & 0.27 & -0.01 & 0.37 \\
\hline
\end{tabular}

$* P<.05 . ; * * P<.01$; Abbreviation: Grip, grip dynamometry.

\begin{tabular}{|c|c|c|c|c|}
\hline & & No difficulty & Some difficulty & A lot of difficulty \\
\hline 1 & Reaching above head & $\square$ & $\square$ & $\square$ \\
\hline 2 & Carrying grocery bags & $\square$ & $\square$ & $\square$ \\
\hline 3 & Using cutlery & $\square$ & $\square$ & $\square$ \\
\hline 4 & Tying shoelaces & $\square$ & $\square$ & $\square$ \\
\hline 5 & Brushing teeth & $\square$ & $\square$ & $\square$ \\
\hline 6 & Clipping nails & $\square$ & $\square$ & $\square$ \\
\hline 7 & Buttoning shirts & $\square$ & $\square$ & $\square$ \\
\hline 8 & Tearing paper & $\square$ & $\square$ & $\square$ \\
\hline 9 & Typing & $\square$ & $\square$ & $\square$ \\
\hline 10 & Writing & $\square$ & $\square$ & $\square$ \\
\hline 11 & Standing from low, seated position & $\square$ & $\square$ & $\square$ \\
\hline 12 & Walking on uneven surface & $\square$ & $\square$ & $\square$ \\
\hline 13 & Climbing stairs & $\square$ & $\square$ & $\square$ \\
\hline 14 & Eating at a normal pace & $\square$ & $\square$ & $\square$ \\
\hline 15 & Swallowing (i.e. choking) & $\square$ & $\square$ & $\square$ \\
\hline 16 & Breathing & $\square$ & $\square$ & $\square$ \\
\hline
\end{tabular}

How many years have you had difficulty swallowing? How many years have you experienced weakness?
TABLE 2 Spearman correlation test, correlation coefficients

TABLE 3 DMFS How much difficulty do you experience with any of the following activities? 
Dimensionality is explored by principal component analysis of fit residuals from fit statistics. ${ }^{38}$

\section{3 | RESULTS}

A total of 20 patients, 7 male and 13 female, ages 20-64 years (median, 29 years; interquartile range [IQR], 27-39) participated in the study (Table S1). Eighteen of 20 (90\%) of patients had renal transplant. Sixteen of $20(80 \%)$ of patients took a form of oral cysteamine. In the initial survey, 12 of 20 (60\%) of patients reported some degree of difficulty swallowing, mainly slow eating and occasional choking and 4 patients with frequent near choking episodes. Seventeen of $20(85 \%)$ of patients reported some degree of (limb) muscle weakness, primarily difficulty using hands, dropping objects and buttoning.

On clinical examination, patients had weakness in neck flexion, shoulder abduction, elbow flexion and extension, wrist flexion and extension, hip flexion, ankle dorsiflexion, and intrinsic hand and foot muscles. The degree of muscle weakness ranged from none to mild involvement of intrinsic hand muscles to prominent distal upper and lower extremity weakness, muscle atrophy, and contractures. The most severely affected proximal muscle weakness was in neck flexion, shoulder abduction, and hip flexion. The most severely affected distal muscles were predominantly the thenar and hypothenar muscles. Seventeen of 20 of the patients had distal upper extremity atrophy and weakness and two of these patients also had distal lower extremity weakness. Three of 20 of the patients had contractures in hand muscle. There were $8 / 20$ patients with proximal upper extremity weakness and 4 of these patients also had proximal weakness in their lower extremities. All patients with proximal weakness also had distal weakness. One patient did not report any muscle weakness in the survey but was found to have mild objective hip and shoulder weakness. One patient-reported mild upper and lower extremity weakness in the survey but had full strength in the clinical examination.

VFSS evaluation showed evidence of dysphagia in 6 of 20 (30\%) of the patients. There was no entry of material into trachea or larynx in 17 patients. The PAS scores ranged from 1 to 8 , with a score of 1 indicating that no material enters into the airway and a score of 8 indicating that material enters the airway, passes the level of the vocal cords and no effort is made to eject. Material contacted the vocal cord without clearing the larynx (PAS 5) in one patient, and material entered the trachea without attempt to clear (PAS 8) in two patients. MEP ranged from 12 to $156 \mathrm{cmH}^{2} \mathrm{O}$, and cough airflow ranged from $98-400 \mathrm{~L} / \mathrm{min}$ (Table 1). The 9-HPT scores ranged from 14.3 to $46.9 \mathrm{~s}$, with higher scores reflecting more severe dysfunction. Patients with dysphagia in VFSS examination did not have significantly different strength compared with patients with normal swallowing function, measured by TUG test, 25-FW, grip dynamometry, EAT-10, or MEP $(P>.05)$. MDADI score was significantly lower in patients with dysphagia in VFSS examination (mean difference 20.4, 95\% Cl (16.3;24.5), $P=.03$ ).
There were significant large correlations between MEP and 25-FW, grip dynamometry and 25-FW, TUG and 25-FW, TUG and MEP, TUG and PF max, and MDADI and EAT-10 (Table 2).

\subsection{Rasch analysis}

Overall, the initial 22-item survey met most expectations of RPCM.

Considering face validity of items, misfitting and psychometrically redundant items were removed and a more practical 16-item survey was developed (Table 3). Thirty-one patients were independently contacted and completed the revised survey. We repeated Rasch analysis on 31 surveys (Figure 1). All items fit the RPCM (Figure 1, horizontal axis), with fit mean square between 0.7 and 1.6. Items "breathing" and

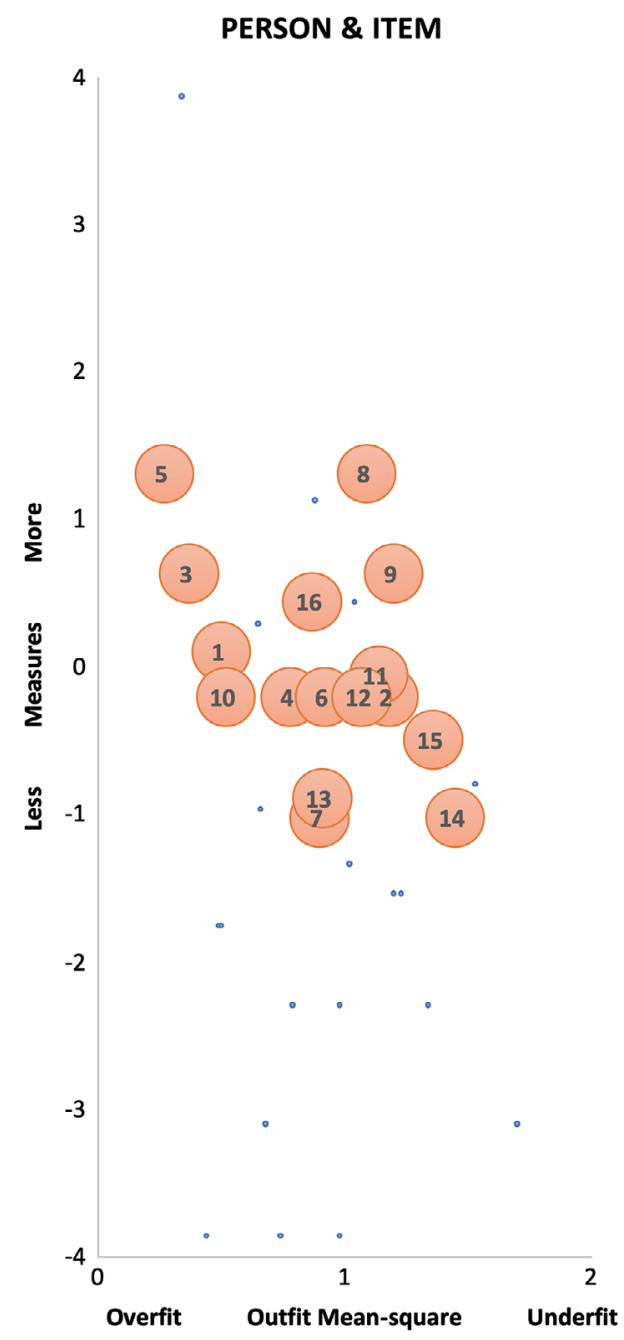

FIGURE 1 Pathway graph maps out items (circles) and patients (dots) on a common logarithmic scale. Item difficulty-Person ability is mapped on the vertical axis with items more appropriate items for more severe disease near the top and easier items near the bottom of the graph. This allows visualizing which items are most useful in discriminating severity at different levels of overall disease severity. The horizontal axis maps how well the items fit under one construct. The item's proximity to the midline on the horizontal axis represents how well the item contributes to what the construct intends to measure [Color figure can be viewed at wileyonlinelibrary.com] 
"standing from low seat" were more frequently scored higher in patients with higher total survey score, suggesting more sensitivity in more symptomatic patients. Items "cutlery" and "buttoning" were scored more often in patients with less total survey score and were likely more sensitive to changes in mildly affected patients. Items pertaining to distal upper extremity function, "cutlery" and "buttoning" formed a minor subdimension (eigen value $3.7743,11.8 \%$ of total unexplained variance) which did not violate the overall unidimensionality of the outcome. Sample size was limited for further analysis of between group differences and differential item functioning (ie, to explore response pattern in patients not taking cysteamine or with different mutations).

\section{4 | DISCUSSION}

Muscle weakness and dysphagia are common in adult patients with nephropathic cystinosis. ${ }^{18,21,22}$ Most of our participants had distal upper extremity weakness measured by manual muscle testing and grip dynamometry. Some participants had more significant weakness, distal muscle atrophy, and contractures. Proximal muscle weakness was primarily limited to neck flexion and shoulder weakness, and two patients had facial muscle weakness. This is in line with previous electrodiagnostic studies of patients with cystinosis where possible myopathic changes (increased insertional activity in the form of fibrillation potentials, positive sharp waves, full interference pattern with a low to normal amplitude, and pathological motor unit action potentials with a reduced amplitude, brief duration, or polyphasic potentials) were noted in patients with no apparent weakness. ${ }^{21}$ While most participants endorsed a certain degree of difficulty swallowing, the more objective swallow testing picked up dysphagia only in three patients. This may suggest that the traditional scoring of more objective testing lacks the sensitivity to capture dysphagia in patients with nephropathic cystinosis. ${ }^{36}$ Similar to other myopathies, a more detailed examination of VFSS studies for kinematic, temporal, and functional measures of swallowing could help further characterize underlying pathophysiology of dysphagia in this patient population. ${ }^{41}$

Despite cysteamine treatment, our patients had weakness and dysphagia. This may suggest that pathophysiology is multifactorial and not solely due to cystine accumulation. While nonadherence and issues related to drug absorption and metabolism may play some role, it is also possible that there may be a secondary metabolic process affecting muscle tissue. ${ }^{42-45}$ In addition, patients on immunosuppressive regiments following renal transplant may develop secondary toxic myopathy and neuropathy. ${ }^{46,47}$ The other less likely possibility is that the muscle tissue may lose its regenerating properties following initial degeneration due to cystine deposition. ${ }^{48}$ Further studies may aid in better characterizing underlying pathophysiology and identifying potential interventional paths.

While exact pathophysiology and dynamics of dysphagia are not entirely well characterized in patients with nephropathic cystinosis, dysphagia has been speculated to be primarily due to underlying myopathic process. $^{21,22}$ Longitudinal evaluations may help better characterize disease progression and identify disease predictors and patients at higher risk.

An important unmet gap in the field of slowly progressive neurodegenerative disorders such as nephropathic cystinosis is the lack of robust biomarkers or patient-reported outcome measures sensitive to capture meaningful changes in disease severity, affecting required sample size and length of treatment trials. Clinical outcomes and biomarkers often lack granularity to capture meaningful changes in disease severity in similar neuromuscular conditions. ${ }^{49}$ For example, a minor change in elbow flexion strength may lead to significant decline in patients' ability to feed themselves independently and have major functional and quality of life impact while less granular manual muscle testing lacks sensitivity to capture such changes in strength.

On the other hand, physiological and imaging biomarkers may have higher noise to signal ratio and less reliability and construct validity. ${ }^{50}$ Learning from our experience with a phenotypically similar condition, $\mathrm{IBM},{ }^{33}$ where the IBM-FRS served as a more sensitive and responsive outcome measure, we developed a disease-specific functional outcome measure and showed better psychometric properties in evaluation of changes in disease severity. Despite some limitations with item targeting (likely reflective of a relatively small sample size), we were able to demonstrate relatively good internal construct validity in terms of reliability, sensitivity, stability, and uni-dimensionality. Depending on clinical trial design, the DMFS may serve as a reliable primary or secondary patient-reported outcome measure in evaluation of patients with nephropathic cystinosis.

These data must be interpreted in the context of the study design. Similar to other rare neurodegenerative studies, the major limitation of our study was a very low sample size for both conventional and psychometric analysis. Despite otherwise acceptable psychometric properties, DMFS has a significant ceiling effect (40\%) likely reflective of a very small sample size (Figure 1, vertical axis). Amalgamating and collapsing scales may help improve item targeting and ceiling effect but may also hinder outcome's responsiveness. Longitudinal evaluation in a larger patient population would help further evaluate its psychometric characteristics and "responsiveness".

\section{ACKNOWLEDGMENTS}

We thank the adult cystinosis patients that contributed to the development of the Cystinosis Adult Care Excellence Initiative project, as well as the patients that participated in this initial study. We would also like to acknowledge valuable input from Minnie Sarwal, MD, PhD.

\section{ETHICAL PUBLICATION STATEMENT}

We confirm that we have read the Journal's position on issues involved in ethical publication and affirm that this report is consistent with those guidelines.

\section{ORCID}

Reza Sadjadi (D) https://orcid.org/0000-0003-3829-6030 


\section{REFERENCES}

1. Ballantyne AO, Trauner DA. Neurobehavioral consequences of a genetic metabolic disorder: visual processing deficits in infantile nephropathic cystinosis. Neuropsychiatry Neuropsychol Behav Neurol. 2000; 13(4):254-263.

2. Kalatzis V, Cohen-Solal L, Cordier B, et al. Identification of 14 novel CTNS mutations and characterization of seven splice site mutations associated with cystinosis. Hum Mutat. 2002;20(6):439-446.

3. Touchman JW, Anikster Y, Dietrich NL, et al. The genomic region encompassing the nephropathic cystinosis gene (CTNS): complete sequencing of a 200-kb segment and discovery of a novel gene within the common cystinosis-causing deletion. Genome Res. 2000;10(2): 165-173.

4. Gahl WA, Bashan N, Tietze F, Bernardini I, Schulman JD. Cystine transport is defective in isolated leukocyte lysosomes from patients with cystinosis. Science. 1982;217(4566):1263-1265.

5. Jonas AJ, Smith ML, Schneider JA. ATP-dependent lysosomal cystine efflux is defective in cystinosis. J Biol Chem. 1982;257(22): 13185-13188.

6. Gahl WA, Reed GF, Thoene JG, et al. Cysteamine therapy for children with nephropathic cystinosis. N Engl J Med. 1987;316(16):971-977.

7. Gahl WA. Cystinosis coming of age. Adv Pediatr. 1986;33:95-126.

8. Gahl WA, Thoene JG, Schneider JA. Cystinosis. N Engl J Med. 2002; 347(2):111-121.

9. Dent CE. The amino-aciduria in Fanconi syndrome. A study making extensive use of techniques based on paper partition chromatography. Biochem J. 1947;41(2):240-253.

10. Malekzadeh MH, Neustein HB, Schneider JA, et al. Cadaver renal transplantation in children with cystinosis. Am J Med. 1977;63(4): 525-533.

11. Thoene JG, Oshima RG, Crawhall JC, Olson DL, Schneider JA. Cystinosis. Intracellular cystine depletion by aminothiols in vitro and in vivo. J Clin Invest. 1976;58(1):180-189.

12. Gretz N, Manz F, Augustin R, et al. Survival time in cystinosis. A collaborative study. Proc Eur Dial Transplant Assoc. 1983;19: 582-589.

13. Pisoni RL, Thoene JG, Christensen HN. Detection and characterization of carrier-mediated cationic amino acid transport in lysosomes of normal and cystinotic human fibroblasts. Role in therapeutic cystine removal? J Biol Chem. 1985;260(8):4791-4798.

14. Kimonis VE, Troendle J, Rose SR, Yang ML, Markello TC, Gahl WA. Effects of early cysteamine therapy on thyroid function and growth in nephropathic cystinosis. J Clin Endocrinol Metab. 1995;80(11): 3257-3261.

15. Markello TC, Bernardini IM, Gahl WA. Improved renal function in children with cystinosis treated with cysteamine. N Engl J Med. 1993; 328(16):1157-1162.

16. Gahl WA, Charnas L, Markello TC, Bernardini I, Ishak KG, Dalakas MC. Parenchymal organ cystine depletion with long-term cysteamine therapy. Biochem Med Metab Biol. 1992;48(3):275-285.

17. Gahl WA, Balog JZ, Kleta R. Nephropathic cystinosis in adults: natural history and effects of oral cysteamine therapy. Ann Intern Med. 2007; 147(4):242-250.

18. Sonies BC, Almajid P, Kleta R, Bernardini I, Gahl WA. Swallowing dysfunction in 101 patients with nephropathic cystinosis: benefit of long-term cysteamine therapy. Medicine (Baltimore). 2005;84(3): 137-146.

19. Charnas LR, Luciano CA, Dalakas M, et al. Distal vacuolar myopathy in nephropathic cystinosis. Ann Neurol. 1994;35(2):181-188.

20. Gahl WA, Dalakas MC, Charnas L, et al. Myopathy and cystine storage in muscles in a patient with nephropathic cystinosis. N Engl J Med. 1988;319(22):1461-1464.

21. Vester U, Schubert M, Offner G, Brodehl J. Distal myopathy in nephropathic cystinosis. Pediatr Nephrol. 2000;14(1):36-38.
22. Sonies BC, Ekman EF, Andersson $\mathrm{HC}$, et al. Swallowing dysfunction in nephropathic cystinosis. N Engl J Med. 1990;323(9):565-570.

23. Nesterova G, Gahl W. Nephropathic cystinosis: late complications of a multisystemic disease. Pediatr Nephrol. 2008;23(6):863-878.

24. Muller-Felber W, Schroder M, Hirschmann M, Kastrup K, Topfer M, Pongratz D. Neurophysiological testing in long-standing cystinosis. Electromyogr Clin Neurophysiol. 1999;39(2):67-70.

25. Trauner DA, Fahmy RF, Mishler DA. Oral motor dysfunction and feeding difficulties in nephropathic cystinosis. Pediatr Neurol. 2001;24(5):365-368.

26. Anikster $\mathrm{Y}$, Lacbawan $\mathrm{F}$, Brantly $\mathrm{M}$, et al. Pulmonary dysfunction in adults with nephropathic cystinosis. Chest. 2001;119(2):394-401.

27. Doyle M, Hammond S, Hammond C, et al. Cystinosis Adult Care Excellence Initiative. Executive summary: initial survey results and recommendations. Cystinosis Research Network. 2016. https://www.cystinosis. org/wp-content/uploads/2019/01/Exec_summary__1_.pdf. Accessed October 9, 2019.

28. Doyle M, Werner-Lin A. That eagle covering me: transitioning and connected autonomy for emerging adults with cystinosis. Pediatr Nephrol. 2015;30(2):281-291.

29. Langman CB, Barshop BA, Deschenes G, et al. Controversies and research agenda in nephropathic cystinosis: conclusions from a "Kidney Disease: Improving Global Outcomes" (KDIGO) Controversies Conference. Kidney Int. 2016;89(6):1192-1203.

30. Doyle M, Werner-Lin A. Family strategies for living with rare disease: the experience of cystinosis. J Soc Social Work Res. 2016;7(3): 547-567.

31. Chen AY, Frankowski R, Bishop-Leone J, et al. The development and validation of a dysphagia-specific quality-of-life questionnaire for patients with head and neck cancer: the M. D. Anderson dysphagia inventory. Arch Otolaryngol Head Neck Surg. 2001;127(7):870-876.

32. Belafsky PC, Mouadeb DA, Rees CJ, et al. Validity and reliability of the Eating Assessment Tool (EAT-10). Ann Otol Rhinol Laryngol. 2008; 117(12):919-924.

33. Cortese A, Machado P, Morrow J, et al. Longitudinal observational study of sporadic inclusion body myositis: implications for clinical trials. Neuromuscul Disord. 2013;23(5):404-412.

34. Slota C, Bevans M, Yang L, Shrader J, Joe G, Carrillo N. Patient reported outcomes in GNE myopathy: incorporating a valid assessment of physical function in a rare disease. Disabil Rehabil. 2018;40(10):1206-1213.

35. Woudt L, Di Capua GA, Krahn M, et al. Toward an objective measure of functional disability in dysferlinopathy. Muscle Nerve. 2016;53(1): 49-57.

36. Rosenbek JC, Robbins JA, Roecker EB, Coyle JL, Wood JL. A penetration-aspiration scale. Dysphagia. 1996;11(2):93-98.

37. Cohen J. Statistical power analysis for the behavioral sciences. Hillsdale, NJ: L. Erlbaum Associates; 1988. xxi, 567 p.

38. Bond TG, Fox CM. Applying the Rasch model: fundamental measurement in the human sciences. Mahwah, NJ: L. Erlbaum; 2001. xxiv, $255 \mathrm{p}$.

39. Narayanaswami P, Burns TM. Clinical outcome assessments: the "Rasch-lonale" for improved accuracy. Muscle Nerve. 2018;58(3): 327-329.

40. Tennant A, Conaghan PG. The Rasch measurement model in rheumatology: what is it and why use it? When should it be applied, and what should one look for in a Rasch paper? Arthritis Rheum. 2007;57(8): 1358-1362.

41. Azola A, Chung T, Mulheren R, Mckeon G, Christopher-Stine L, Palmer J. Dysphagia in inflammatory myositis: a study of the structural and physiologic changes resulting in disordered swallowing. In: ACR/ARHP Annual Meeting, San Diego, CA, 2017. Abstract 2147.

42. Cherqui S, Sevin C, Hamard G, et al. Intralysosomal cystine accumulation in mice lacking cystinosin, the protein defective in cystinosis. Mol Cell Biol. 2002;22(21):7622-7632. 
43. Guicciardi ME, Leist M, Gores GJ. Lysosomes in cell death. Oncogene. 2004;23(16):2881-2890.

44. Kalatzis V, Antignac C. Cystinosis: from gene to disease. Nephrol Dial Transplant. 2002;17(11):1883-1886.

45. Racusen LC, Wilson PD, Hartz PA, Fivush BA, Burrow CR. Renal proximal tubular epithelium from patients with nephropathic cystinosis: immortalized cell lines as in vitro model systems. Kidney Int. 1995;48 (2):536-543.

46. Galindo M, Cabello A, Joven B, et al. Mycophenolate mofetil induced myopathy in a patient with lupus nephritis. J Rheumatol. 2005;32(1):188-190.

47. Rush DN. Cyclosporine toxicity to organs other than the kidney. Clin Biochem. 1991;24(1):101-105.

48. Loell I, Lundberg IE. Can muscle regeneration fail in chronic inflammation: a weakness in inflammatory myopathies? J Intern Med. 2011;269 (3):243-257.

49. Lunn MP, Van den Bergh PY. Outcome measures in neuromuscular disease: is the world still flat? J Peripher Nerv Syst. 2015;20(3): 255-259.
50. Froeling M, Nederveen AJ, Nicolay K, Strijkers GJ. DTI of human skeletal muscle: the effects of diffusion encoding parameters, signal-tonoise ratio and T2 on tensor indices and fiber tracts. NMR Biomed. 2013;26(11):1339-1352.

\section{SUPPORTING INFORMATION}

Additional supporting information may be found online in the Supporting Information section at the end of this article.

How to cite this article: Sadjadi R, Sullivan S, Grant N, et al. Clinical myopathy in patients with nephropathic cystinosis.

Muscle Nerve. 2020;61:74-80. https://doi.org/10.1002/mus. $\underline{26726}$ 